\title{
PROINSULIN IN HEALTHY PREGNANCY, PREGNANCY WITH GESTATIONAL DIABETES AND AFTER DELIVERY
}

\author{
M. P. Genova ${ }^{1}$, K. Todorova-Ananieva ${ }^{2}$, B. Atanasova ${ }^{1}$ and K. Tzatchev ${ }^{1}$ \\ ${ }^{1}$ Department of Clinical Laboratory and Clinical Immunology, Medical University, \\ University Hospital "Alexandrovska", Sofia, Bulgaria \\ 2University Clinic of Endocrinology, Medical University, Pleven, Bulgaria
}

Summary. The aim of the present study was to evaluate the levels of pro-insulin and pro-insulin/ insulin ratio (PIR) in pregnant with normal glucose tolerance (NGT), pregnant with gestational diabetes mellitus (GDM) and women after delivery with GDM history. Normal pregnancy is characterized by progressive insulin resistance, which is physiologically compensated by an increase in insulin secretion. The higher secretion of the insulin precursor pro-insulin has been associated with $\beta$-cell dysfunction. A total of 102 pregnant women between 24-28 gestational weeks (53 GDM pregnant, 49 with NGT) and 22 post partum with GDM history, as assessed by a $2 \mathrm{~h}$ oral glucose tolerance test, were included in the study. Fasting plasma insulin and pro-insulin (PI) concentrations at the basal state were measured in all women. The ratio pro-insulin/insulin was calculated. BMI was significantly higher in GDM pregnant compared to NGT weight-matched group (30.56 \pm 6.9 vs. $30.56 \pm 6.9 ; p<0,011)$ and compared to the levels after delivery $(30.56 \pm$ $6.9 v s$. $27.9 \pm 6,27 ; p<0,001)$. Significant differences in the levels of $\mathrm{PI}$ between NGT and GDM pregnant ( $3.94 \pm 2.78$ vs. $7.59 \pm 5.27 ; p=0.006)$, between GDM and post partum women ( $7.59 \pm 5.27$ vs. $4.46 \pm 1.14 ; p=0.022)$ were established. No significant difference in the level of PIR between two pregnant groups was observed. Separately NGT and GDM showed significant difference compared to young mothers $(0.41 \pm 0.14$ vs. $0.148 \pm 0.031, p<0.02 ; 0.46 \pm 0.16$ vs. $0.148 \pm$ $0.031, p=0.009$ ). Fasting insulin was statistically higher in GDM pregnant compare to NGT and women after delivery $(13.84 \pm 8.43$ vs. $11.35 \pm 7.38, p=0.02$; $13.84 \pm 8.43$ vs. $10.60 \pm 7.53, p<0.01)$. The correlation between PIR and BMI in the three groups studied were $r=0.416 ; r=0,741 ; r=0,556$ (with statistical significance $p=0.01$ between NGT and GDM pregnancy, $p=0.02$ between GDM pregnancy and post-partum, $p<0.0001$ between NGT pregnancy and young mother with GDM history). In our study, comparison of PI levels between pregnant with 
NGT and GDM demonstrated that the OR of developing GDM was $1.194(95 \% \mathrm{Cl}$, $1.028-1.329, P=0.001)$. Increasing the value of $\mathrm{PI}$ with $10 \mathrm{pmol} / \mathrm{l}$ increases the risk for development of GDM with $19.4 \%$. According to our results, pregnant with GDM have elevated levels of pro-insulin and PIR which could serve as a markers for this condition. These results support our findings about relationship and influence of BMI on $\beta$-cell functions, established in this study with normotolerant, gestational diabetes pregnant and women post partum with GDM history. These results demonstrate that gestational diabetics have abnormalities in pancreatic beta-cell secretion, which are likely to be important both in the etiology of gestational diabetes and non-insulin dependent diabetes.

Key words: body mass index, gestational diabetes mellitus, pro-insulin, PIR

\section{INTRODUCTION}

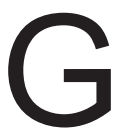
estational diabetes mellitus (GDM) is defined as any degree of glucose intolerance with onset or first recognition during pregnancy [2]. GDM appears to result from the same broad spectrum of physiological and genetic abnormalities that characterize diabetes without pregnancy. Indeed, women with GDM are at high risk for developing diabetes later in the life [7].

Gestational diabetes mellitus (GDM), like type 2 diabetes (type $2 \mathrm{DM}$ ), is characterized by metabolic defects of insulin resistance and relative insufficiency of insulin secretion [4]. GDM is a form of hyperglycemia that results from an insulin supply inadequate to meet the tissue demands for normal blood glucose regulation [11]. Women with GDM tend to be obese; adipose tissue affect carbohydrate metabolism affecting different metabolic pathways.

Progressive $\beta$-cells dysfunction has been reported in women with GDM that, over years, leads to progressive hyperglycemia and diabetes after pregnancy [21]. GDM appears when the increase of insulin secretion is not enough to compensate for the increased insulin resistance in pregnant women [7, 21]. Pro-insulin levels increase together with insulin concentrations in insulin resistance [17], a raised PIR, due to a disproportionate release of pro-insulin from $\beta$-cells, is considered an early marker of islet dysfunction [5].

The aim of the present study was to study the levels of serum pro-insulin and PIR in women suffering from gestational diabetes mellitus, evaluate any change or reversibility of serum pro-insulin or PIR during the postpartum period and the impact of $\mathrm{BMI}$ on these processes.

\section{MATERIAL AND METHODS}

A total of 102 pregnant women between 21-28 gestational weeks (53 with GDM and 49 with normal glucose tolerance) and 22 postpartum women (8-14 weeks) were included in the study. We assessed selected anthropometric, clinical, and 
pathophysiological parameters in all of them. Exclusion criteria for pregnant women were chronic diseases, acute infection during pregnancy or establishing of diagnosis, drugs affected the carbohydrate metabolism or interfered with insulin sensitivity, multiple pregnancies, known diabetes, fetal malformation, or other severe maternal illnesses, age $<18$ or $>45$ years. Informed consent has been obtained in written from all patients.

All the tested pregnant women with no previously diagnosed diabetes went through screening for gestational diabetes mellitus with a $2 \mathrm{~h} 75 \mathrm{~g}$ oral glucose tolerance test (OGTT). Diagnosis of GDM was in accordance with the recommendations of the International Diabetes in Pregnancy Study Group - fasting plasma glucose $\geq 5.1$ $\mathrm{mmol} / \mathrm{L}, 1 \mathrm{~h} \geq 10.0 \mathrm{mmol} / \mathrm{L}, 2 \mathrm{~h} \geq 8.5 \mathrm{mmol} / \mathrm{L}$ [12]. The OGTT stratified participants into two glucose tolerance groups: normal glucose tolerance (NGT, $n=49)$ and gestational diabetes mellitus (GDM, $n=53$ ). All women after delivery with a history of GDM went through screening with $2 \mathrm{~h} 75 \mathrm{~g}$ oral glucose tolerance test too $(n=22)$.

The following data were collected for all women: age, pregnancy BMI at GDM diagnosis, BMI after delivery, gestational weeks, weeks postpartum. Samples were drawn just before, at 60 and 120 min after ingestion of glucose for GDM diagnosis and after birth.

Blood samples for insulin, glucose and pro-insulin measurements were drawn from individuals in fasting state between 8.00 am and 9.00 am, after a 12-hours fasting pause overnight. Plasma glucose was determined in venous blood by the method of oxygen consumption (Analox GM9, Analox Instruments USA, reference range 2.8-6.1 mmol/L). Serum insulin concentration in venous blood was analysed by electrochemiluminescent immunoassay (ECLIA) (Elecsys 2010, Roshe Diagnostics, reference range $2.6-24.9 \mu \mathrm{U} / \mathrm{ml}$ ). Serum for pro-insulin was frozen at $-20^{\circ} \mathrm{C}$ and has been done using the ELISA method (Mercodia, AB Company, Uppsala, Sweden, reference range 3,3-28 pmol/l). Internal Quality Control Scheme in applied by control materials "Control L" 10.4 (8.55-12.3 pmol/l) and "Control H, 40.8 (34.7-46.9 pmol/l). The measurement is performed on ELISA-reader "Zenith 3100" on company "Anthos". All laboratory assays were performed at Central Clinical Laboratory, University Hospital "Alexandrovska".

The self-reported weight is expressed as kilograms $(\mathrm{kg})$ and the height measured during the interview is expressed as squared meters $(\mathrm{m} 2)$ to calculate maternal BMI $\left(\mathrm{kg} / \mathrm{m}^{2}\right)$.

\section{Statistical analysis}

Data were analyzed using Statistical software for Windows 13.0 by SPSS SPSS. The Shapiro-Wilk test was used to determine whether each variable had a normal distribution. These variables were expressed as means \pm SD. The KruskalWallis test and the $U$ Mann-Whitney test were used to compare selected groups. A value $p<0.05$ value was defined as significant. Comparisons between the subgroups were performed by one-way analysis of variance (ANOVA) with post-hoc analysis to locate the differences. 


\section{RESULTS}

A total of 102 pregnant women were enrolled in the study (mean age $31.5, \mathrm{SD} \pm$ 4.55 years) and divided in two main groups based on the plasma glucose concentration and the IAPSDG criteria for diagnosis of GDM. Women in the NGT group were younger than those in the GDM group and postpartum with statistically significant difference $(p<0.003, p<0.01)$. BMI was significantly higher in GDM pregnancy compared to NGT and women post partum ( $<0.011$; $p<0.001)$. Meanwhile BMI was higher in women after delivery compare to NGT $(p<0,0001)$ (Table 1). Three groups were comparable regarding the fasting glucose, glucose at $60 \mathrm{~min}$, at 120 min, fasting insulin and pro-insulin, and PIR (Table 2).

Table 1. Clinical characteristics of the participants

\begin{tabular}{|l|l|l|l|l|}
\hline Characteristics & $\begin{array}{l}\text { NGT group } \\
\left(\mathrm{n}_{1}=49\right)\end{array}$ & $\begin{array}{l}\text { GDM group } \\
\left(\mathrm{n}_{2}=53\right)\end{array}$ & $\begin{array}{l}\text { After delivery } \\
\left(\mathrm{n}_{3}=22\right)\end{array}$ & $\begin{array}{l}\text { Statistical } \\
\text { significance }\end{array}$ \\
\hline Age (years) & ${ }^{*} 0,51 \pm 4,72 \cdot$ & $* 32.6 \pm 4,39$ & $\bullet 33.6 \pm 4.3$ & $\begin{array}{l}\mathrm{P}<0.003^{*} \\
\mathrm{p}<0.01 \cdot \bullet\end{array}$ \\
\hline BMI $\left(\mathrm{kg} / \mathrm{m}^{2}\right)$ & ${ }^{*} 26.83 \pm 5,54^{*}$ & $\cdot 30.56 \pm 6.9^{*}$ & $\cdot 27.9 \pm 6,27^{* *}$ & $\begin{array}{l}\mathrm{p}<0,011^{*} \\
p<0,001 \cdot \\
<0,0001^{* *}\end{array}$ \\
\hline Gestational weeks & $24 \pm 4$ & $24 \pm 4$ & - & NS \\
\hline
\end{tabular}

GDM - gestational diabetes mellitus; BMI - body mass index. Values of parameters are presented as mean \pm SD or count (percentage)

During pregnancy, the mean plasma glucose levels during $75 \mathrm{~g}$ OGTT were significantly higher in women with gestational diabetes compared with normal glucose tolerant pregnant women at the fasting state, 60 and 120 minutes $(p<0.0001 ; p<$ $0.001 ; p<0.005)$. After delivery, the mean plasma glucose levels were significantly lower in women after birth at 60 minutes and 120 min than the GDM women ( $p=$ $0.002 ; p<0.0001)$.

The mean values of fasting insulin levels were significantly higher in GDM women as compared to NGT pregnant and women after birth $(p=0.02 ; p<0.01)$ and significant difference between NGT pregnant and after delivery was observed ( $p$ $<0.01$ ). During pregnancy the mean values of pro-insulin were significantly higher GDM women than the NGT group $(p=0.006)$ without significant difference for PIR. After delivery pro-insulin and PIR were significantly lower in comparison to GDM women ( $p=0.022 ; p=0.009$ ) (Table 2). A significant positive relation was found between BMI and the PIR in the GDM women, normal glucose tolerant pregnant as well as in the women post partum $(r=0.416, p=0.01 ; 0.741, p=0.02 ; 0.556$, $\mathrm{p}<0.001$ ) (Table 3). 
Table 2. Characteristics of the patients' metabolic parameters

\begin{tabular}{|c|c|c|c|c|}
\hline Characteristics & $\begin{array}{l}\text { Pregnant with NGT } \\
\qquad\left(n_{1}=49\right)\end{array}$ & $\begin{array}{c}\text { Pregnant with GDM } \\
\left(n_{2}=53\right)\end{array}$ & $\begin{array}{l}\text { After delivery } \\
\qquad\left(n_{3}=22\right)\end{array}$ & $\begin{array}{l}\text { Statistical } \\
\text { significance }\end{array}$ \\
\hline $\begin{array}{l}\text { Fasting glucose } \\
(\mathrm{mmol} / \mathrm{l})\end{array}$ & $\cdot 4.62 \pm 0.28^{\star}$ & $5.93 \pm 1.04^{*}$ & $\cdot 5.33 \pm 0.79$ & $\begin{array}{l}p<0,0001^{*} \\
p<0.0001 .\end{array}$ \\
\hline $\begin{array}{l}\text { Glucose at } 60 \mathrm{~min} \\
(\mathrm{mmol} / \mathrm{l})\end{array}$ & ${ }^{*} 6.95 \pm 1.37^{\star *}$ & $+8.49 \pm 2.29^{* *}$ & ${ }^{*} 7.62 \pm 2.36+$ & $\begin{array}{l}p<0.001^{* *} \\
p=0.0004^{*} \\
p=0.002+\end{array}$ \\
\hline $\begin{array}{l}\text { Glucose at } 120 \mathrm{~min} \\
(\mathrm{mmol} / \mathrm{l})\end{array}$ & ${ }^{*} 6.14 \pm 1.21 \dagger$ & $\cdot 7.49 \pm 2.04 \dagger$ & $\cdot 7.62 \pm 2.09^{*}$ & $\begin{array}{l}p<0.005 \dagger \\
p<0.0001 . \\
p<0.0001^{*}\end{array}$ \\
\hline Fasting insulin (mlU/l) & $* 11.35 \pm 4.98^{* *}$ & $\cdot \bullet 13.84 \pm 8.43^{\star *}$ & $\bullet \cdot 10.6 \pm 7.53^{*}$ & $\begin{array}{l}p=0.02^{* *} \\
p<0.01 \bullet \\
p<0.01^{*}\end{array}$ \\
\hline $\begin{array}{l}\text { Pro-insulin at } 0 \mathrm{~min} \\
\mathrm{pmol} / \mathrm{l}\end{array}$ & $3.94 \pm 2.78^{*}$ & $\star \star 7.59 \pm 5.27^{\star}$ & ** $4.46 \pm 1.14$ & $\begin{array}{l}p=0.006^{*} \\
p=0.022^{* *}\end{array}$ \\
\hline $\begin{array}{l}\text { Pro-insulin/insulin ratio } \\
\text { (PIR) }\end{array}$ & $0.41 \pm 0.14 \ddagger \ddagger$ & $0.46 \pm 0.16 \ddagger$ & $\ddagger \ddagger 0.148 \pm 0.031 \ddagger$ & $\begin{array}{l}p=0.009 \ddagger \\
p<0.02 \ddagger \ddagger\end{array}$ \\
\hline
\end{tabular}

Table 3. Correlation between BMI and PIR in the three studied groups

\begin{tabular}{|c|c|c|c|}
\hline & $\begin{array}{c}\text { Pregnant with NGT } \\
\left(n_{1}=49\right)\end{array}$ & $\begin{array}{c}\text { Pregnant with GDM } \\
\left(n_{2}=53\right)\end{array}$ & $\begin{array}{c}\text { After delivery } \\
\left(n_{3}=22\right)\end{array}$ \\
\cline { 2 - 4 } & BMl & BMl & BMl \\
\hline Pro-insulin/insulin ratio (PIR) & 0,416 & 0,741 & 0,556 \\
& $p=0.01$ & $p=0.02$ & $p<0.001$ \\
\hline
\end{tabular}

\section{DISCUSSION}

In normal pregnancies, the basal need of insulin can be almost double [11] and this increase is relevant by its permanent character. Over it will be superimposed the daily physiologic postprandial secretory insulin demand. In this context, the "functional pancreatic reserve" will be exceeded in a low percentage of cases (1-2\%) which is equal with the prevalence of GDM in normal weight population [16]. It is widely accepted now that insulin resistance and hyper- insulinemia are characteristic features of late pregnancy and that gestational diabetes is associated with a failure in insulin secretory capacity to compensate for insulin resistance. Gestational diabetes mellitus is one of the most common complications of pregnancy [7] and frequently predictive of later maternal impaired glucose tolerance or type 2 diabetes mellitus [19].

In the present study, the pregnant women presented a metabolic pattern suggestive of enhanced insulin resistance, namely increased fasting insulin levels. It was demonstrated as the mean values of plasma insulin during pregnancy which was significantly higher in GDM women compared with the NGT group and women after delivery. 
Homko et al. [11] studied the insulin secretion during late gestation (third trimester) and postpartum. Insulin resistance remained higher in women with GDM. They concluded that the women with GDM had a major $\beta$-cell defect with impossibility to compensate for their increased level of insulin resistance, which occurred during late pregnancy.

Based on data accumulated during the last years, it can be stated that irrespectively of the type of diabetes, the primary and probably the main $\beta$-cell change could be identified in the increase of the pro-insulin levels and of PIR [13].

It becomes evident that one of the primary $\beta$-cell defect in the processing of pro-insulin can be seen evolved in one of two distinct but parallel pathways: the pro-insulin/insulin defect can explain the increased levels of plasma pro-insulin, that is associated automatically with decreased plasma insulin levels as well as with the mentioned defects of the insulin-secretory pattern. Increased plasma proinsulin levels could be seen as a common denominator for all the diabetes phenotype, including the condition GDM [13]. Rentnakaran (2005) established that the relative $\mathrm{PI}$ secretion in late pregnancy is primarily associated with insulin resistance and it is in related to positive PIR, but do not always reflects $\beta$-cell function. It looks likely that women with a history of GDM could develop relative hyperproinsulinemia for a long time in tandem with progressive $\beta$-cell dysfunction thus leading to the development of T2DM [18].

The important feature in the results of our study is that the mean levels of pro-insulin were significantly higher during pregnancy in GDM pregnant than NGT and after delivery. Another important observation is the mean values of PIR during pregnancy and after delivery. This ratio was not significantly higher in GDM women in comparison to NGT, but significantly higher, compared to women after delivery. In this group women with GDM history the PIR was significantly lower, compared to PIR in NGT. The PIR could be a better marker in assessing the dysfunction of pancreatic $\beta$-cells in the early period after birth [3].

Previous studies of relative pro-insulin levels in GDM have yielded conflicting results. Some earlier studies signaled the presence of increased pro-insulin and PIR in gestational diabetes compared with the NGT controls [15]. Kautzky-Willer and et al. [15] found that both serum pro-insulin and PIR were significantly higher in GDM patients compared with control subjects. They also reported that after delivery the PIR remained elevated. Increased pro-insulin concentrations and a raised PIR are specific for GDM and might thus serve as its marker and potentially even identify subjects at high risk for the development of type 2 diabetes. In contrast, Hanson and et al. [10] did not support the hypothesis that an increased fasting PIR is a marker for later development of type 2 diabetes or impaired glucose tolerance in former GDM subjects. Swinn and et al [20] studied the relationship between abnormalities of glucose metabolism and the 32, 33 split pro-insulin. They reported elevated levels of such pro-insulin in GDM patients both fasting and 120-min versus pregnant normoglycemic control subjects. 
Furthermore, F. et al. [9] investigated the levels of pro-insulin, specific insulin $(\mathrm{Spl})$ and the $\mathrm{PI} / \mathrm{Spl}$ ratio in consecutive pregnant women with normal glucose tolerance, and with GDM, in comparison to healthy, non-pregnant women. They found no significant differences in the levels of $\mathrm{PI}$ and the $\mathrm{PI} / \mathrm{Spl}$ ratio between pregnant and non pregnant women, and between pregnant women with GDM and NGT. So, they suggested that in normoglycemic pregnancy as well as GDM pregnancy metabolic alterations including enhanced insulin resistance and hyperglycemia, did not result in increased circulating levels of specific insulin, as indicated by PIR. Several factors may have contributed to the lack of consistent findings in these studies. These factors include differences among the study populations with respect to severity of glucose intolerance, body weight, and hepatic insulin extraction. Finally, description of the basal PIR is useful for characterising the $\beta$-cell status [20].

More recent studies, reported by Amara et al., established that serum levels of pro-insulin and the PIR were significantly higher in women with GDM than the control and reference groups during pregnancy and after delivery $(p=0.0001)$ [1]. The increased plasma pro-insulin could be better explained by a supplementary beta cell load due to the supplementary body fat mass which will stimulate the production of a higher amount of insulin [13].

The content of fat in the human body has its important biological significance, well expressed during pregnancy. Pro-insulin was positively correlated with BMI [6]. In this connection, the relative $\mathrm{PI}$ secretion was assessed in this study by using of PIR. In our study was assessed the impact of BMI on pro-insulin-insulin ratio. It was found a high positive correlation between PIR and BMI in the three studied groups $r=0.416 ; r=0,741 ; r=0,556$ for NGT, GDM and post partum women, respectively, with statistical significance $p=0.01$ between NGT and GDM pregnancy, $p=0.02$ between GDM pregnancy and post-partum, $p<0.0001$ between NGT pregnancy and young mother with GDM history.

In conclusion, we established that pregnant with GDM have elevated levels of pro-insulin and PIR which could serve as a markers for this condition. These results support our findings about relationship and influence of BMI on $\beta$-cell functions, established in this study with NGT, GDM pregnant and women post partum with GDM history. Our data demonstrate that gestational diabetics have abnormalities in pancreatic $\beta$-cell secretion, which are likely to be important both in the etiology of gestational diabetes and non-insulin dependent diabetes. We think that a mix of hormones, their interplay with cytokines and the insulin signaling pathway seem to be responsible for the metabolic changes occurring during pregnancy. Individual genetic background and additional factors, in particular obesity, determined the ability of a pregnant women to balance these changes and to remain a physiological milieu. 


\section{REFERENCES}

1. A mara, F. E. et al. Study of pro-insulin level and its role in a cohort of women with gestational diabetes in Alexandria, Egypt. - J. Diabetology, 2011, 1-3, http://www.journalofdiabetology.org/

2. American Diabetes Association. Diagnosis and classification of diabetes mellitus. - Diabetes Care, 30, 2007, S42-S47.

3. Andy, M. et C. Ionescu-Tirgoviste. Fetal pro-insulin and insulin and placental weight in pregnancies complicated by gestational diabetes. - Research, 8, 2012, № 29, 140-145.

4. B en-Harous h, A, Y. Yogev et M. Hod. Epidemiology of gestational diabetes mellitus and its association with Type 2 diabetes. - Diabet. Med., 21, 2004, № 2, 103-133.

5. B ergman, R. N., D. T. Finegood et S. E. Kahn. The evolution of $\beta$-cell dysfunction and insulin resistance in type 2 diabetes. - Eur. J. Clin. Invest., 32, 2002, (Suppl. 3), 35-45.

6. B i r k e l a n d, K. I. et al. Hyperpro-insulinaemia of type II diabetes is not present before the development of hyperglycemia. - Diabetes Care, 17, 1994, 1307-1310.

7. Buchanan, T. A. et A. H. Xiang. Gestational diabetes mellitus. - J. Clin. Invest., 115, 2005, 485- 491.

8. Dornhorst, A. et al. Abnormalities in fasting circulating proinsulin concentration in mild gestational diabetes. - Clin. Endocrinol. (Oxf), 34, 1991, № 3, 211-213.

9. Festa, A., N. Shnawa, G. Schernthaner et S. M. Haffner. Pro-insulin in pregnant women with normal glucose tolerance or mild gestational diabetes mellitus. - Exp. Clin. Endocrinol. Diabetes, 107, 1999, 447-453.

10. Ha n s o n, U., B. Persson, S. G. Hartling et C. Binder. Increased molar pro-insulin-to-insulin ratio in women with previous gestational diabetes does not predict later impairment of glucose tolerance. Diabetes Care, 19, 1996, № 1, 17-20.

11. Hom ko, C. et al. Insulin secretion during and after pregnancy in patients with gestational diabetes mellitus. - J. Clin. Endocrinol. Metab., 86, 2001, 568-573.

12. Internation al Association of Diabetes and Pregnancy Study Groups Consensus Panel 2010. Diabetes Care, 33, 2010, № 3, 676-682.

13. I onescu-Tîggovişte, C. et C. Guja. Pro-insulin, pro-amylin and the beta-cell endoplasmic reticulum: the key for the pathogenesis of different diabetes phenotypes.

14. Kautzky-Willer, A. et A. Bancher-Todesca. Diabetology in pregnancy. - Basel, Karger, 2005, 18-33.

15. Kautzky-Willer, A. et al. Elevated islet amyloid pancreatic polypeptide and pro-insulin in lean gestational diabetes. - Diabetes, 46, 1997, 607-614.

16. Pas cu, M., J. A. Marin, C. Guja et C. Ionescu-Tirgoviste. Pro-insulin level in women with gestational diabetes according to bodi mass index. - Proc. Rom. Acad. Series B, 2, 2011, 117-123.

17. Mykkänen, L. et al.. The relation of pro-insulin, insulin, and pro-insulin-to-insulin ratio to insulin sensitivity and acute insulin response in normoglycemic subjects. - Diabetes, 46, 1997, 1990-1995.

18. Rentnakaran, R., A. Hanley, M. Sermer et B. Zinman. The impact of insulin resistance on proinsulin secretion in pregnancy: hyperproinsulinemis is not a featute of gestational diabetes. - Diabetes care, 28, 2005, № 11, 2710-2715. 
19. S obngwi, E. et al. Effect of a diabetic environment in utero on predisposition to type 2 diabetes. - Lancet, 361, 2003, 1861-1865.

20. Swinn, R. A. et al. Excessive secretion of insulin precursors characterizes and predicts gestational diabetes. - Diabetes, 44, 1995, 911-915.

21. Xi a n g, A. H. et al. Detailed physiological characterization of the development of type 2 diabetes in Hispanic women with prior gestational diabetes mellitus. - Diabetes, 59, 2010, 2625-2630.

$\equiv$ Corresponding author:

Mariana P. Genova, MD, PhD

Department of Clinical Laboratory and Clinical Immunology Medical University Hospital "Alexandrovska"

1 Sv. Georgy Sofiyski st.

Bg -1431 Sofia

e-mail: mariana8sofia@yahoo.com 